\title{
Método de interpolação aplicado à espacialização de chuvas para o município de Jaguaretama-Ce
}

\author{
Interpolation method applied to the spatial distribution of rainfall for the city of \\ Jaguaretama- Ce \\ $\operatorname{Dantas}^{1}$, S. P. \\ sulivandantas@yahoo.com.br
}

\begin{abstract}
Resumo
A utilização de software no tratamento de dados para análises em pesquisas da Geografia está cada vez mais ganhando espaço devido a sua contribuição quanto a sistematização de dados primários e secundários. Nesse contexto, tomamos para esse trabalho o método de interpolação como subsídio de representação da espacialização de precipitação para o município de Jaguaretama/CE, tendo uma série temporal de quinze anos para esse trabalho. Através do programa Sufer 10.0, com o uso do método de Krigagem foi possível estimar a representação espacial das chuvas durante o período de análise, favorecendo assim, a caracterização da precipitação da área de estudo.
\end{abstract}

Palavras-chave: Espacialização, Precipitação, Semiárido.

\begin{abstract}
The use of software in processing data for analysis in Geography research is increasingly gaining ground because of its contribution as the systematization of primary and secondary data. In this context we take for this work the interpolation method as precipitation spatial representation allowance for the municipality of Jaguaretama / EC with a time series of fifteen years for this work. Through Sufer 10.0 program, using the Kriging method was possible to estimate the spatial distribution of rainfall during the analysis period, thus promoting the characterization of the precipitation of the study area.
\end{abstract}

\section{INTRODUÇÃO}

Agregar valores encontrados numa determinada área através de uma planilha de dados com coordenadas $\mathrm{x}$, y e z; por meio de uma coordenada gera-se um ponto de referência. O conjunto de pontos é interpolado resultando gráficos com curvas delimitando zonas. Essa, talvez seja a principal função do método de interpolação utilizado.

Para Souza et.al. (2011), a interpolação é um procedimento que permite construir um novo conjunto de dados a partir de um conjunto discreto de dados pontuais conhecidos, ou seja, trata-se de um método que possibilita proceder à reconstituição (aproximada) de uma função apenas conhecendo algumas das suas abscissas e respectivas ordenadas (imagens). Assim, em suma, a interpolação constitui-se num método de estimar um parâmetro para o qual não existe informação disponível, considerando-se que os pontos próximos no espaço tendem a ter valores mais semelhantes do que pontos mais afastados.

Segundo Miranda (2005), o processo de interpolação é constituído de duas partes, sendo a primeira a definição de um relacionamento de vizinhança, e o segundo, a definição de qual método calculará os valores desconhecidos. 
O objetivo deste trabalho é espacializar a precipitação pluviométrica do município de Jaguaretama (CE) tomando como base de dados os postos pluviométricos de localidades internas ao território e municípios vizinhos.

\section{METODOLOGIA}

\section{1. Área de estudo}

O município de Jaguaretama está localizado na porção centro-leste do Estado do Ceará. Localiza-se a uma latitude de $5^{\circ} 36^{\prime} 46^{\prime \prime}$ Sul e uma longitude de $38^{\circ} 46^{\prime} 01^{\prime \prime}$ Oeste, estando a uma altitude de $100 \mathrm{~m}$.

Tem como municípios limítrofes: a Norte, Morada Nova e Banabuiú; a Sul: Solonópole, Jaguaribe e Jaguaribara (antiga, atualmente barragem do açude Castanhão); a Leste: Jaguaribara, Morada Nova e Alto Santo e; a Oeste: Banabuiú e Solonópoles. O município de Jaguaretama está a aproximadamente $240 \mathrm{~km}$ da capital cearense. Na figura 01 consta a localização do Município de Jaguaretama em relação ao Estado do Ceará, com destaque a presença do açude Padre Cícero (Castanhão).

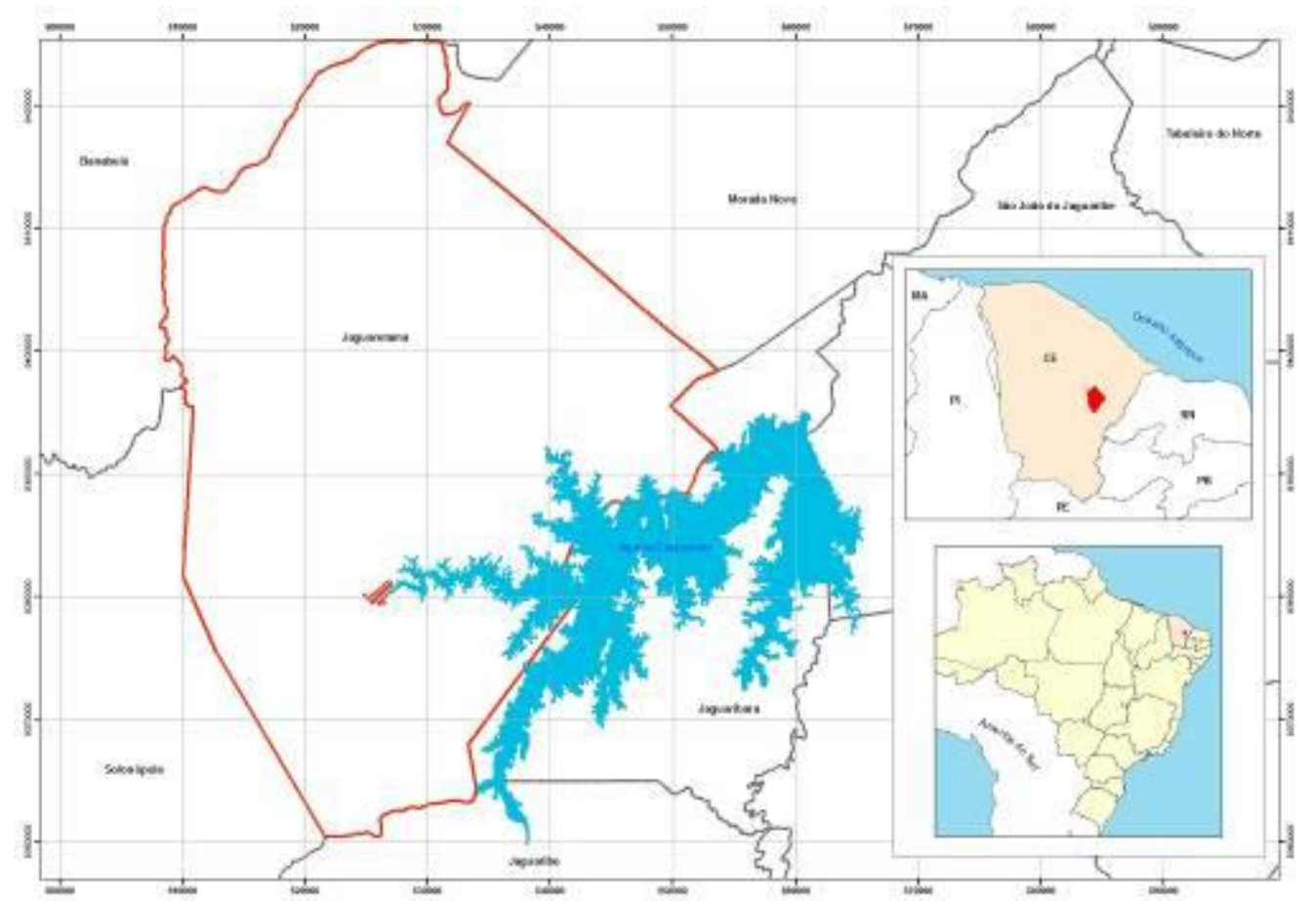

Figura 01: Localização do município de Jaguaretama (CE).

Jaguaretama faz parte do chamado sertão centro-oeste do Estado do Ceará, este município inserido no semiárido cearense com características físicas geográficos de irregularidades climáticas, solos rasos, depressão sertaneja, abastecido pelo sistema hídrico de açudagem. 
O município tem seu período mais quente no segundo semestre do ano, justamente por serem meses mais secos devido à estiagem no Estado do Ceará. Apresentando médias máximas anuais de $28,0^{\circ} \mathrm{C}$.

Para Guerra e Vasconcelos (2005) devido à combinação desses sistemas com os fatores geográficos, tais como latitude, orientação do litoral em relação à corrente dos alísios, as baixas altitudes, o relevo, a orientação das serras, a dimensão continental e o posicionamento do seu território em relação ao hemisfério sul, caracteriza as condições climáticas vigentes no estado do Ceará. Sendo caracterizado por elevadas temperaturas, baixos índices de nebulosidade, forte insolação, elevadas taxas de evaporação e marcante irregularidade das chuvas no tempo e no espaço, principal característica do seu regime pluviométrico.

O município situa-se no domínio hidrogeológico das rochas cristalinas, de baixo potencial, A esse fato está associado às condições climáticas provocando graves problemas ligados ao abastecimento de água. Quanto a Geomorfologia do município, essa é caracterizada por Depressão Sertaneja submetida a processos de sedimentação, que propiciam o aumento e a predominância de criações (bovinos, ovino-caprinucultura, pequenos animais, etc.), com topografia local levemente acidentada.

Segundo Guerra e Vasconcelos (2005) o município de Jaguaretama está inserido nas Bacias do Rio Banabuiú e do médio Jaguaribe. Como principais drenagens superficiais, podem-se mencionar os riachos Timbaúba, das Pedras, Fundo e do Sangue, merecendo destaque ainda os riachos do Ferreira, dos Cavalos, da Cruz, desterro e do Livramento, Santa Rosa e Santana, o rio Banabuiú, onde faz divisa com município de mesmo nome.

Devido a levantamento feito, em 2007, pelo Instituto de Pesquisa e Estratégia Econômica do Ceará (IPECE) é possível afirmar que o Município conta com potencial hídrico considerável, tendo em vista ser banhado por rios perenes e além de açudes públicos e particulares de médio porte, bem como pela Barragem do Castanhão que banha 1/5 das terras rurais do Município, inclusive, banhando também parte da zona urbana.

\subsection{Procedimentos operacionais}

- Levantamentos bibliográficos, cartográficos e visitas técnicas nos órgãos públicos do município de Jaguaretama;

- Dados pluviométricos de postos meteorológicos monitorados pela Funceme, disponibilizados pela Agência Nacional de Águas - ANA (site hidroweb);

- Software para interpolar os dados, Surfer 10;

- Software Excel 2007 para tabulação dos dados. 
A representação de dados no espaço é essencial em diversas áreas do conhecimento, sendo de uso extensivo em agronomia, biologia, ecologia, geografia, geologia, meteorologia, etc (LANDIM, 2002). O uso de métodos de interpolação espacial de dados está cada vez mais disseminado nos estudos geográficos. A partir do levantamento bibliográfico realizado, foi elencado o modelo interpolador de análise de dados espaciais que mais se adequou ao intuito desse trabalho, a saber: Krigagem.

Por meio do SURFER 10.0 realizou-se a interpolação adotando o método de Krigagem. Esse é um pacote de programas gráfico comercial desenvolvido pela Golden Software Inc. que é utilizado para o cálculo e a confecção de mapas variáveis a partir de dados regularmente distribuídos.

Com a rede de postos pluviométricos monitorados pela Fundação Cearense de Meteorologia e Recursos Hídricos (Funceme) disponíveis para o município de Jaguaretama/CE e municípios vizinhos foi possível realizar a interpolação dos dados. Apesar da dificuldade encontrada quanto à ausência de dados e a grande quantidade de falhas existentes nas séries disponibilizadas, foram escolhidos as melhores séries de dados dos postos pluviométricos.

Os dados foram organizados (Tabela 01) em uma planilha estatística e processados, com o intuito de calcular os valores médios da pluviosidade e homogeneizá-los. Apesar da indicação da Organização Mundial de Meteorologia, de utilizar dados mínimos de uma série de 30 anos para estudos em Climatologia, para esse trabalho foi possível organizar dados de 12 anos (2004 a 2015) devido à ausência de dados para a área de estudo e da região do entorno, considerando assim uma definição de série provisória.

Tabela 01: Dados dos postos pluviométricos organizados pelo autor.

\begin{tabular}{lccccc}
\hline Posto Pluviométrico & Responsável & Altitude(m) & X & Y & Média(mm) \\
\hline A - Jaguaretama & FUNCEME & 150 & 9390216 & 527690,9 & 879 \\
B - Jaguaretama & FUNCEME & 150 & 9378794 & 525471,2 & 879,4 \\
C - Serrote Branco & FUNCEME & 214 & 9393905 & 512923,1 & 691,2 \\
D - Solonópole & FUNCEME & 170 & 9369956 & 498154,1 & 876,8 \\
E - Jaguaribara & FUNCEME & 89 & 9375469 & 542450 & 703,9 \\
F - Jaguaribe & FUNCEME & 120 & 9347833 & 542431,4 & 732 \\
G - Morada Nova & FUNCEME & 89 & 9436245 & 570198,7 & 672,5 \\
\hline
\end{tabular}

Posterior tabulação dos dados, esses foram espacializados (Figura 02), tomando como base as coordenadas geográficas dos postos pluviométricos utilizados, essas coordenadas também estão disponíveis no banco de informações da Funceme. Após a espacialização dos postos, foi possível realizar a interpolação dos dados de pluviometria estimada para o município de Jaguaretama/CE. 


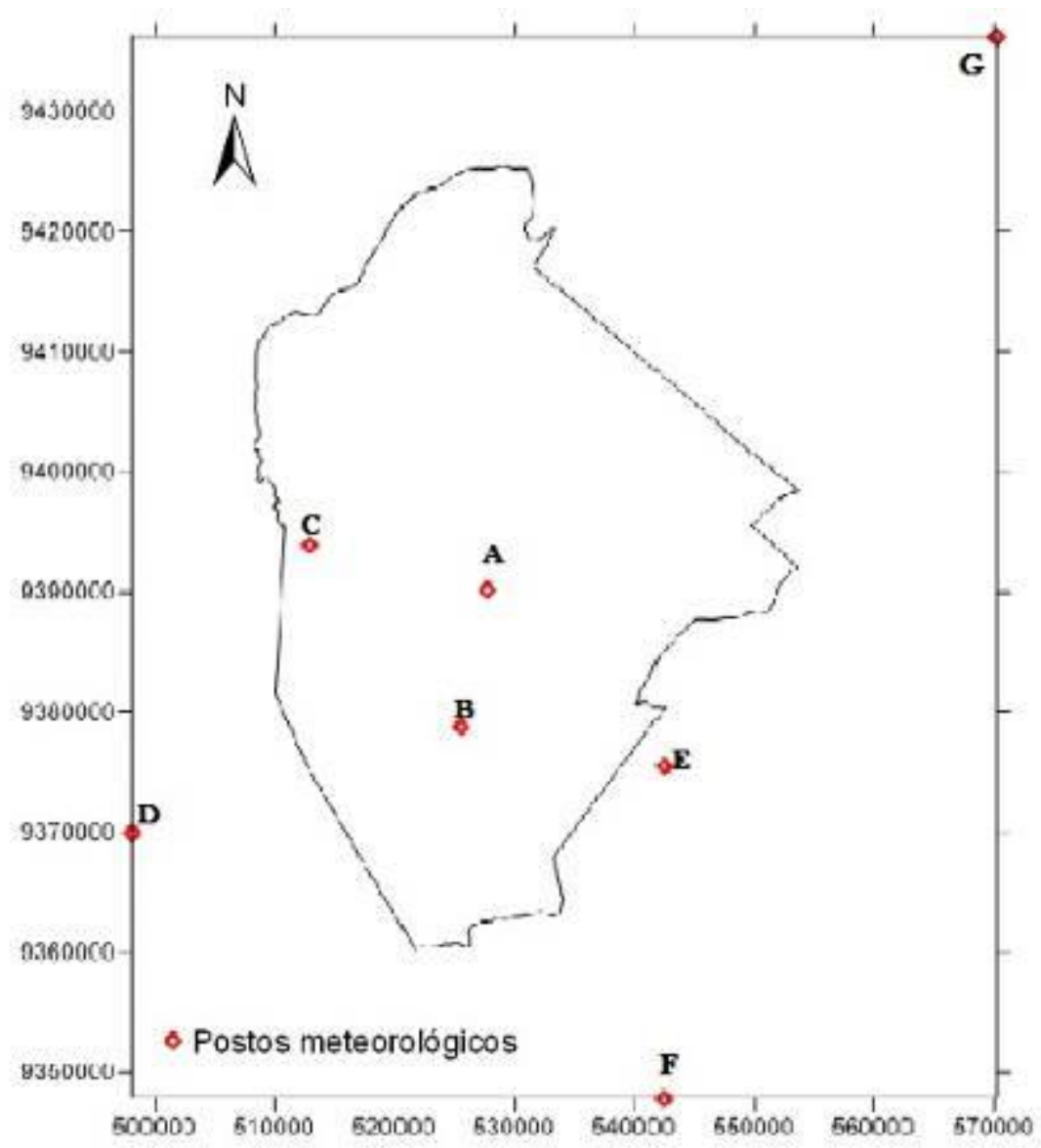

Figura 02: Espacialização dos postos pluviométricos de Jaguaretama/CE e de seu entorno.

\section{RESULTADOS E DISCUSSÃO}

Souza (2011) destaca que os interpoladores podem também variar quanto à transição (abrupta ou gradual), ao seu caráter (determinístico ou probabilístico) e quanto à exatidão, sendo caracterizados como exatos ou inexatos, neste sentido, destaca-se que interpoladores exatos respeitam os dados existentes, enquanto os inexatos ou aproximados assumem incertezas (erros) nos dados existentes.

Nesse estudo de caso, esse caráter probabilístico teve uma boa aceitação quanto à interpolação de dados pluviométricos para a região. Pois, como mostrado na figura 3, a concentração de chuvas apresentou maiores valores para as altitudes mais elevadas da região, sendo o setor oeste de Jaguaretama.

Por esse motivo acredita-se que o modelo por Krigagem seja o mais adequado, pois no tocante a espacialização do fenômeno pluviométrico, este modelo se apresenta como o mais apto a representação, possibilitando a geração da carta de isoieta com boa fidelidade aos dados amostrados. 
A krigagem é um método de interpolação que se utiliza de geoestatística, este, possui em sua base conceitual dois importantes fundamentos, o das variáveis regionalizadas e das funções aleatórias (MIRANDA, 2005). Para esse trabalho foi utilizado o modelo de Krigagem simples, esse assume a média como estatisticamente constante para toda área.

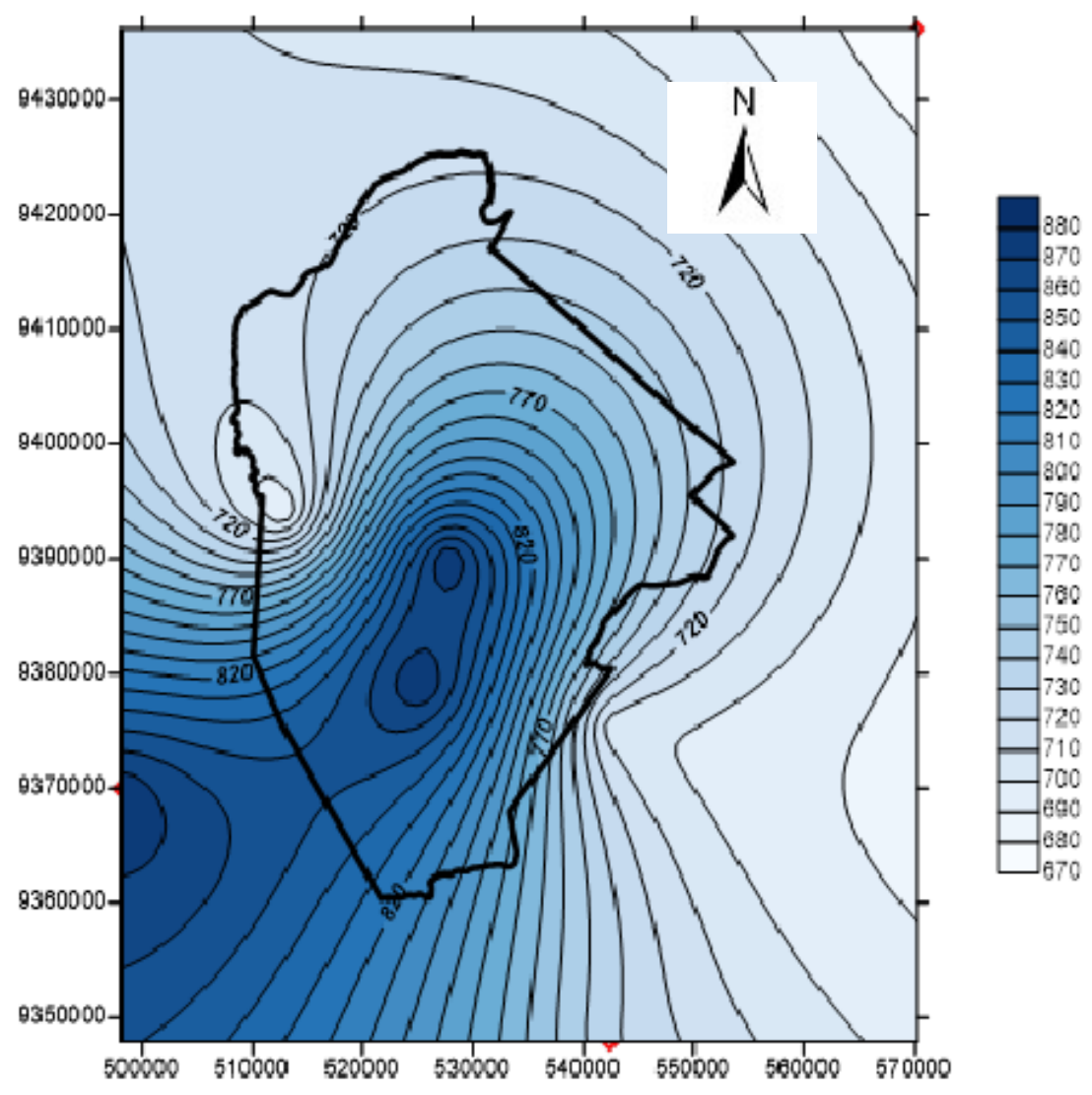

Figura 03: Modelo de espacialização de chuvas gerado pela Krigagem.

Observa-se na figura acima, a concentração de maiores valores por isoietas (mais precisamente na isoieta de $820 \mathrm{~mm}$ ) em total de chuvas para o município é, também, na sede de Jaguaretama. Essa recebe a influência direta do Açude Castanhão, porém não se pode afirmar que esse registro de maior concentração de chuvas próximo ao açude seja em função da presença do lago artificial do reservatório, sendo necessário um estudo mais detalhado sobre os dados de chuvas, além dos demais atributos climáticos.

Observa-se que na figura 04 a altimetria do município de Jaguaretama se apresenta de maneira homogênea, caracterizada por estar localizada na depressão sertaneja e com valores hipsométricos entre $100 \mathrm{~m}$ e $200 \mathrm{~m}$ de altitude. Portanto, vê-se a necessidade de uma análise mais detalhada da distribuição das chuvas no município, pois a altitude, nesse caso, também interfere na dinâmica das chuvas locais. 


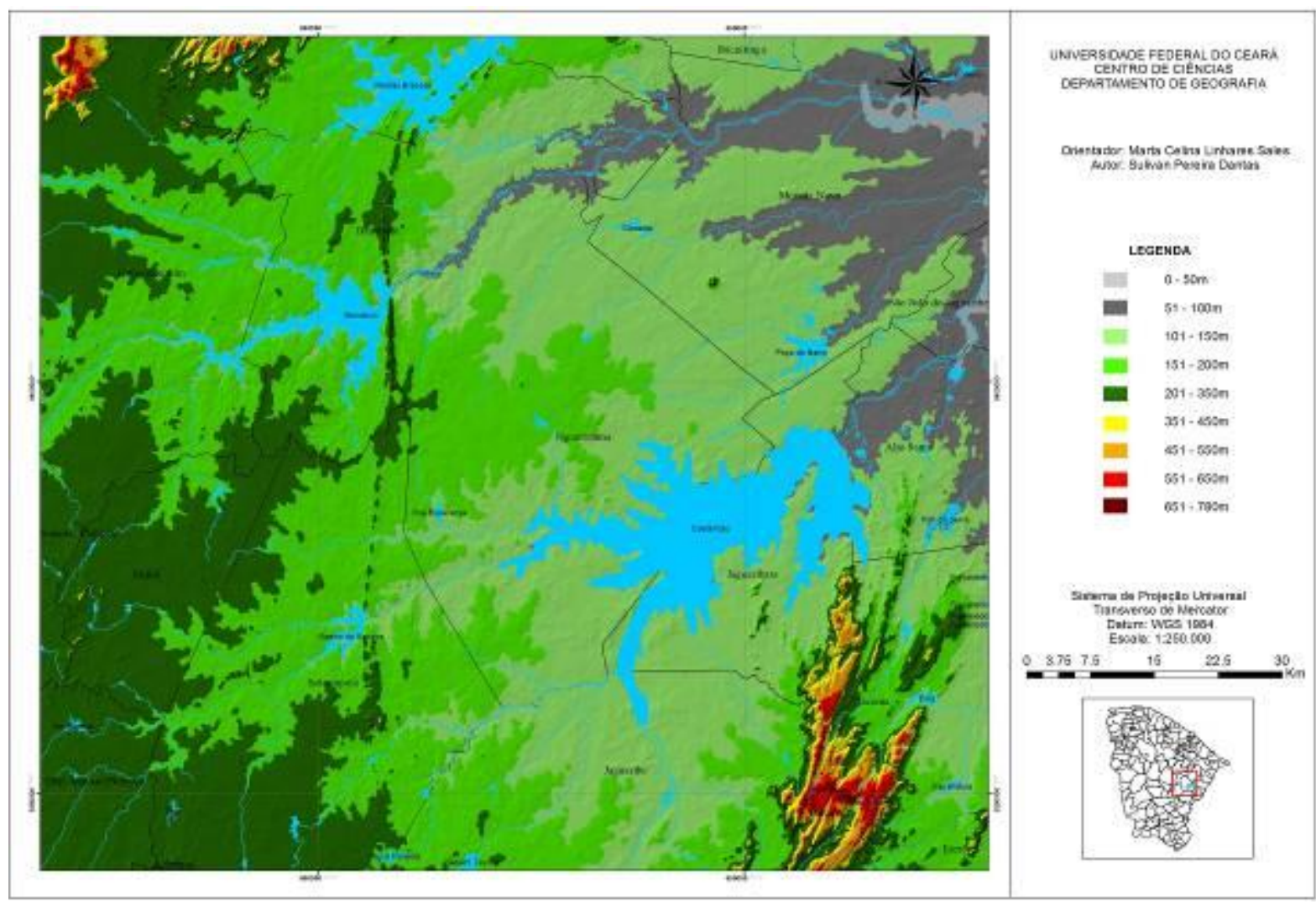

Figura 04: Mapa hipsométrico do município de Jaguaretama-Ce. Fonte: do autor.

Para Mello et al. (2003), o conhecimento da chuva de áreas do projeto é essencial para o êxito de um projeto de engenharia ligado as obras hidráulicas. Para o bom dimensionamento de barragens para contenção do excesso de água e, ou, represamento para fins múltiplos, terraços e bacias de contenção de erosão, drenagem rural e urbana, pontes, canais e outras estruturas hidráulicas, a estimativa mais precisa possível de uma chuva intensa é extremamente importante para que um projeto seja viável tanto do ponto de vista técnico quanto econômico.

Nesse contexto, a espacialização de chuvas para Jaguaretama/CE torne-se essencial, pois esse município é banhado pelo Açude Público Padre Cícero (Castanhão). E esse é o maior em usos múltiplos do Brasil, com capacidade de $6.700 .000 \mathrm{~m}^{3}$. Tendo assim uma maior atenção quanto ao levantamento hidroclimático para a região de grandes projetos de reservatórios, principalmente em ambientes semiáridos, com alta vulnerabilidade socioambiental.

Assim como, ao iniciar um projeto de intervenção hidráulica, como é o caso da construção de grandes barragens, deve-se considerar a importância do Relatório Preliminar Ambiental (RAP) com a finalidade de conhecer a dinâmica ambiental local e adjacente, principalmente quando esse projeto se trata de uma intervenção hidroclimática. Tendo, desta forma, entender que a construção de reservatórios de grande porte nessa área pode contribuir com a alteração sedimentológica do rio represado e das condições climáticas locais, como, o próprio ciclo da água. 


\section{CONSIDERAÇÕES FINAIS}

Diante do resultado apresentado observa-se o grau de importância dessa aplicação de espacialização, nesse caso, da precipitação, pois através da interpolação de dados pluviométricos é possível caracterizar a área de estudo para que haja um planejamento agrícola, gestão de recursos hídricos, intervenção por obras hidráulicas, avaliação e planejamento ambiental, dentre outros.

A utilização de softwares para elaboração de cartogramas contribui de maneira importantíssima para os trabalhos técnicos e acadêmicos, pois através deles é possível projetar intervenções mais conscientes quanto aos impactos gerados.

No caso deste trabalho, verificou-se a contribuição quanto ao método geoestatístico sugerido para trabalhos futuros que tenham como objetivo levantar as características hidroclimáticas locais, as possíveis intervenções com a construção de grandes barragens e as possibilidades metodológicas de obtenção de informações através de softwares livres.

Cabe ressaltar, que por meio do programa Sufer 10.0 é possível elaborar diversos modelos que tenham objetivos relacionados à representação cartográfica, esse se tornou eficaz para o objetivo desse estudo. Além da eficiência, para a espacialização de chuvas, do método de krigagem Simples.

\section{REFERÊNCIAS}

SOUZA, J. L. Leite de. GOMES, T. S. DIAS, R. dos S. OLIVEIRA, M. de A. SANTOS, Rosângela L. Avaliação de métodos de interpolação aplicados à espacialização das chuvas no território identidade Portal do Sertão / Bahia. Anais XV Simpósio Brasileiro de Sensoriamento Remoto SBSR, Curitiba, PR, Brasil, 30 de abril a 05 de maio de 2011, INPE p.4295.

MIRANDA, J. I. EMBRAPA INFORMAÇÃO TECNOLÓGICA. Fundamentos de sistemas de informações geográficas. Brasília: EMBRAPA Informação Tecnológica, 2005.- 425p.

GUERRA, C. B. VASCONCELOS, S; M. S. Aspectos qualitativos das águas subterrâneas do município de Jaguaretama, Ceará - Brasil. Revista de Geologia, Vol. 18, nº 2, 123-129, 2005. IPLANCE - Fundação Instituto de Planejamento do Ceará, 1998, Perfil Básico Municipal: Jaguaretama - CE. Fortaleza, 42p

IPECE - Instituto de Pesquisa e Estratégia Econômica do Ceará, 2007, Relatório de Desempenho: Jaguaretama - CE. Fortaleza, 15p.

LANDIM, P. M. B. MONTEIRO, R. C. Introdução à confecção de mapas pelo software sufer. Departamento de Geologia Aplicada - IGCE. UNESP: 2002. 
MELLO et al. Krigagem e inverso do quadrado da distância para interpolação dos parâmetros da equação de chuvas intensas. R. Bras. Ci. Solo, 2003.

Recebido em: 14/08/2016

Aceito para publicação em: 01/10/2016 